\title{
Creating Adaptive and Individual Personalities in Many Characters Without Hand-Crafting Behaviors
}

\author{
Jennifer Sandercock, Lin Padgham, and Fabio Zambetta \\ School of Computer Science and Information Technology, \\ RMIT University, Melbourne, Australia. \\ \{jennsand, linpa, fabio\}@cs.rmit.edu.au
}

\begin{abstract}
Believable characters significantly increase the immersion of users or players in interactive applications. A key component of believable characters is their personality, which has previously been implemented statically using the time consuming task of hand-crafting individuality for each character. Often personality has been modeled based on theories that assume behavior is the same regardless of situation and environment. This paper presents a simple affective and cognitive framework for interactive entertainment characters that allows adaptation of behavior based on the environment and emotions. Different personalities are reflected in behavior preferences which are generated based on individual experience. An initial version of the framework has been implemented in a simple scenario to explore which parameters have the greatest effect on agent diversity.
\end{abstract}

\section{Introduction}

Computer games and interactive applications for entertainment and training are fast growing areas. Today the aim is no longer solely to create good graphics there are growing demands with regard to the complexity and believability of the virtual characters. Believable characters significantly increase the immersion and the 'fun' that a player has in an interactive application. Typically programmers, designers and animators contribute to hand-crafting the various characters. However this is a time consuming and expensive approach, and not even feasible when large numbers of different characters are needed. Most prior work that aims to create characters with emotions and personality has concentrated on one or two characters (such as [3]) or superficial crowd simulations (such as [2]).

In our work we are investigating models and architectures which allow development of complex and believable characters with minimum effort, using an intelligent agent base, complemented with emotion and personality. Personality has long been seen to be a key aspect of creating believability in virtual characters since it gives them the individuality and quirkiness to make them engaging[12]. Further, personality provides the coherence, or stability that ties together different behavioral choices. We will use the term personality to encompass the individual way that each person reasons and evaluates the world based 
on experience and genetics, which in turn is reflected in behavior and expression. According to Ortony [17] personality can be considered as "a generative engine that contributes to coherence, consistency, and predictability in emotional reactions and responses" [17]. Ideally we want our characters to exhibit individual variability or personality, but for that personality to be coherent, rather than chaotic. Further, we would like to achieve all this as simply as possible.

As well as creating diverse characters, we aim to develop characters that are able to adapt their personality over time. Most implementations of personality are static during execution and are based on trait based theories, such as the popular Five Factor Model[15]. This approach does not, however, provide support for ways in which people express different traits, depending on their current situation. For instance, behavior at a football match is usually very different to the behavior of the same person in a cinema. In Ortony's words, behavioral consistency depends on the environmental situation[17]. Using a fixed personality is often justified by the fact that most people believe our adult personalities are relatively static. However, personality does develop over time, particularly during childhood experiences or when the environment itself changes in a substantial and long term manner. For instance, if subjected to a long period of being abused every time someone speaks to a stranger, even an outgoing person eventually becomes introverted to some degree or in certain situations. It is the development phase of personality that we aim to mimic by allowing the characters to adapt to their environment. Characters that are able to adapt to a player would create a significantly more immersive environment compared to characters that are the same no matter how many times they are ignored, for instance. The challenge is to allow the characters to make consistent decisions based on their current state as well as their past experience and environment.

\subsection{Our Approach}

The approach we take is to use a Belief Desires Intention (BDI) agent platform which supports choices in ways of accomplishing goals and sub-goals, as well as having a robust failure recovery approach. The philosophical foundations of BDI explain rational behavior in terms of concepts such as goals, intentions and commitments, which are modeled explicitly in BDI systems. As discussed in [?] the BDI structure of goals (desires), with multiple plans (intentions) to achieve those goals, which are themselves made up of sub-goal steps, provides enormous possibilities for diversity. We combine this facility for diversity in behavior that can be observed by a player, with a mechanism for modeling emotions and personality, which then influences the choices of each individual agent within this large space of possibilities.

We start with characters that have only minor, and easily achieved, differences. Then, based on the experiences of the characters in the virtual world and their adaptation to that world, we evolve a range of characters with different personalities as expressed in their behavior. Behavior includes anything that the agent is able to do in the environment, for example gestures, actions, conversation, vocal tone. 
The approach we use is most suitable for game genres with longer character interaction times since players need time before complexity is noticeable[13]. Characters in interactive entertainment can take two forms: actors and avatars. Actors are characters that can act of their own free will in the virtual environment; whereas avatars are controlled (at times) by a human player. Our work is concerned with the former. In order to avoid confusion between the terms 'character' and 'agent', we will use the term 'agent' to refer to the reasoning part of a 'character'.

Our model is inspired by Ortony's more recent work [17], where he states that in order to create believable agents with individuality they have to be coherent at a global level, "across different kinds of situations, and over quite long time periods" [17]. We call this form of consistency, personality coherence. We believe there are four reasons for differences within and across individuals, the first three are inspired by [17] and are about internal evaluations of emotions, whereas the last is about the way emotions are managed and expressed:

1. Differences in evaluation and construal of the world (e.g. whether you are winning a football match or not depends on which team you are on; and importance placed on winning affects evaluation);

2. Differences in the way that emotions affect us, called emotionality (e.g some people are more volatile than others);

3. Current state of the individual and their view of the environment;

4. Differences in how one deals with emotions (e.g. does a person act to try and change the world, or do they adjust their aspirations).

In this paper we describe the simplified representations we use for emotion and personality, inspired by, but simplified from, the work of Lazarus [14] (as implemented by Gratch and Marsella [10]) and the work of Ortony [17] as described above. We also describe a simple interactive environment which we are using to explore and test these models. In our current model we are concentrating on using personality to decide on coping (including action) preferences and to influence evaluation.

We begin by presenting our simplified model of how agent behavior is determined by selection of plans to achieve goals, based on current internal (emotional) and external (environmental) states, combined with personality. We then provide a detailed presentation of our representation of emotions, personality and how they are adapted. Following this we describe the analysis of our test-bed application. We finish with a description of related work and a brief description of the planned future directions for this work.

\section{Architectural Framework}

Our cognitive-behavioral architecture in Figure 1 improves character diversity and personality coherence across situations, and relies on coping processes as a key concept. The term coping process refers to any plan that helps the agent deal with its emotions. For instance, coping processes to deal with being scared 
could be running away, or re-considering the situation to realize that there is nothing to be scared about. Our framework has three core processes (shown as the large diamonds in figure 1): appraisal of coping preferences, selection of coping processes and evaluation or re-appraisal.

Appraisal of coping preferences is a mechanism which can be used at key execution points, and is a process by which the agent ranks the different coping processes available to it. Selection of coping processes chooses the actual plan to execute based on preference rankings and plan applicability. This selection process takes into account what plans (or coping processes) are actually viable in the current situation. In the evaluation process the agent reviews the success of its chosen approach. We begin by giving an overview of our framework and a standard agent execution cycle and then describe the processes and entities in more detail.

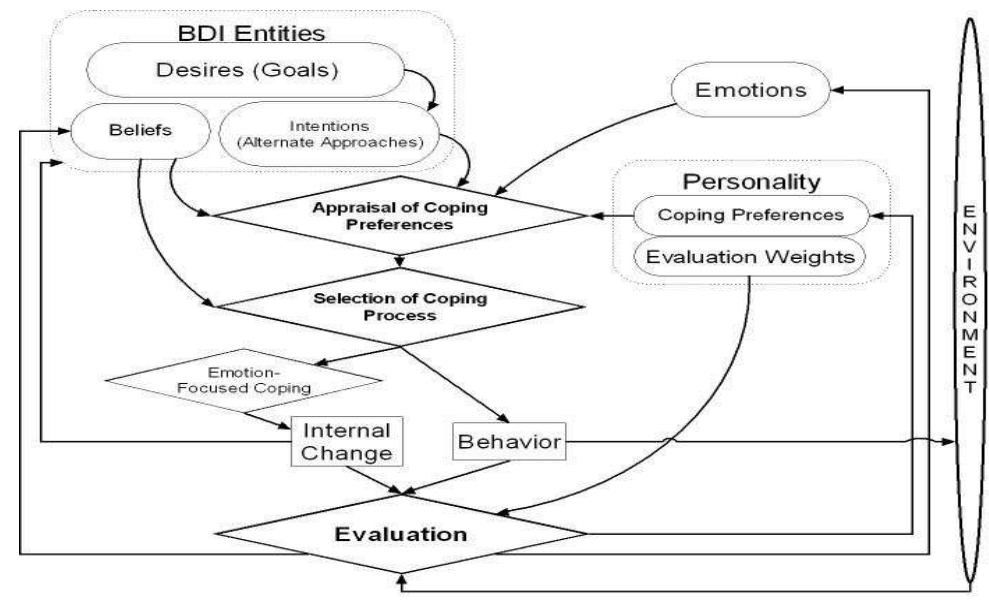

Fig. 1. Cognitive-behavioral Framework.

As seen at the top left of our framework diagram in figure 1, an agent's beliefs, desires, intentions, current emotional state and personality feed into appraisal of coping preferences. These preferences, along with beliefs about the world and the BDI plan selection process, allow coping strategies to be chosen. If the first selected plan fails, then the coping preferences guide subsequent choices. Coping processes can affect behavior of the agent in the virtual environment or effect an internal change to its beliefs, desires and intentions. As seen at the bottom of figure 1, evaluation or re-appraisal can be triggered either by: (i) a change to the external environment, (ii) an internal change, or (iii) behavior that the agent has performed. Agent personality and current emotions of the agent itself and of the other agent(s) in the interaction affect evaluation which in turn updates for future emotions, beliefs and coping preferences (which are part of personality). Changes to personality reflect the agent's adaptation to 
its environment and experience. Our loop of cause and effect means that each aspect of person, environment and behavior can influence any other aspect of the three. This is a core principle of social learning theory [4], although in our model agents adapt behavior based solely on personal experience and not based on observation. The full framework as described above is only used at key execution points which significantly change the agent's behavior. At all other points during execution where the agent is required to make a decision the agent selects its behavior based solely on plan applicability, without the added step of assigning preferences.

The agents begin execution with the same initial emotions, personality, goals and plans, but with different beliefs and emotionality (see section 3 for a description of emotionality). Differences can be specified by the character designer, or randomly ascribed to reduce the designer's workload. In our system we consider there to be two different forms of beliefs: knowledge, perceived 'facts' about the environment and other agents; and opinions, beliefs that are linked to an emotional judgment. Agents are initialized with a small amount of personal knowledge (randomly allocated) and opinions that are independent of the other agents, ie they start with no knowledge or opinions of the other agents. During execution the five aspects of beliefs, desires, intentions, emotions and personality change as the agent interacts with the environment and other agents. It is this that gives the agent its unique identity.

\subsection{Example Using Test-bed Application}

We have implemented our framework in a simple virtual world inhabited by young school children who have never met each other before and who can have conversations with each other. The children have one goal: to become happy. How they achieve this goal is entirely based on their diverse personalities. They are able to form beliefs and opinions about the other agents in the system.

During a full execution cycle the agent begins by finding the plans (intentions) that could achieve its current goals. For example, plans that could help achieve happiness include talking to a friend or re-evaluating the number of friends that you have. The agent's personality, current emotions and current beliefs inform the appraisal process which ranks the preference for each plan, e.g. when the agent is sad and doesn't have many friends it may prefer to find a friend to talk to, or it may want to find someone new to talk to. The agent then attempts to execute plans until it finds one that succeeds, e.g. the agent may have no friends, or none that are able to talk to it currently. A selected plan can either change the agent's behavior in the environment or change its internal beliefs, goals and plans, e.g. talking to someone will directly affect the agent's behavior in the environment, and re-evaluating friends will affect the agent's beliefs. After the plan is completed, the agent evaluates the success of this plan in achieving its goal, e.g did talking to a friend make the agent happier. This evaluation directly affects its own emotions, personality (in the form of allocation of coping preferences) and its opinions of the environment and other agents. For example, if the agent didn't have a 'good' (in its opinion) conversation with its friend 
then it will decrease its opinion of the other agent, its sadness will increase and if the interaction was very bad, then the agent will be less likely to choose that plan for that situation (ie initial state of the environment and the agent) again. Initial plan preferences are allocated randomly and the evaluation phase has a large random component during initialization. So not all agents who initially try the plan 'be by myself' will enjoy it and therefore not all agents are likely to choose that plan again.

\subsection{Implementation of Cognitive Appraisal Model}

Our framework is based on the cognitive appraisal model of affect which maintains that emotions can only be updated or triggered after an appraisal of the world and events. There are many variations on cognitive appraisal, but we follow the model proposed by Lazarus [14] which has two main components: coping and appraisal. Coping is we how we deal with emotional encounters. Appraisal is the process of deciding which form of coping to implement and the process of analyzing changes in the internal and external world of the agent. There are two types of coping: emotion-focused coping and problem-focused coping [14]. Problem-focused coping results in observable behaviors, emotion-focused coping results in changes to beliefs, desires, and intentions. There are also two types of appraisal in our model: appraisal of coping preferences and evaluation or re-appraisal of changes to the external and internal environment of the agent. Both forms of appraisal are influenced (in our framework) based on the agent's personality.

Although the most obvious way to cope is, perhaps, to act in the world (problem-focused coping), an alternative way is to modify one's internal state (emotion-focused coping). A person (or a Sim) who is unhappy because they don't have a car, can work to be able to buy a car, or alternatively they may modify their values so that not having a car is something to be proud of. The emotionfocused coping process in our framework captures this second kind of mechanism and requires an internal reasoning process (rather than simple actions) to occur to change beliefs, desires and intentions. Emotion-focused coping processes include: belief formation and changes; goal formation, adoption, and abandonment; and planning, ie intention modification or generation. Problem-focused coping processes are implemented in our framework as any form of behavior that the agent is able to exhibit in the virtual environment, such as gestures, actions and conversations. Our preliminary implementation uses problem-focused coping only, which can easily be modified to include other coping strategies since the agent adapts based on past experience and not based on hard-coding.

There are two levels to selecting a coping plan, which are represented in figure 1 by the middle two large diamonds. The first is a type of appraisal, $a p$ praisal of coping preferences, which places preferences for some strategies over others based on personality. The second is selection of coping processes which uses beliefs about the environment or the current situation along with the BDI mechanism of context conditions to find applicable plans. The plan with the highest preference according to the initial appraisal process is chosen initially 
by the selection process, as long as it is viable in the current situation. However, if the initially chosen plan fails during execution then BDI goal persistence mechanisms ensure that an alternative (less preferred) plan will be tried if it is applicable in the situation. For example, the agent may prefer to increase its happiness by finding treasure. But if there is no treasure available, and no means to acquire any treasure, a less preferred approach to increasing happiness will be selected, such as finding a job other than treasure hunter.

Our implementation of the two-stage selection process is similar to other models that use an affective phase and then a cognitive phase for the decisionmaking process. These models are usually based on Damasio's somatic-marker hypothesis [6] which states that before we make every decision our bodies automatically focus attention towards or away from some response options (often this process is subconscious). The appraisal of cognitive preferences process is similar to placing relative somatic-markers on plans in order to guide the cognitive process towards those that have an initial preference based on emotions and personality.

The evaluation phase (which is the second type of appraisal) is triggered by a change to the internal or external world of the agent or by something the agent itself has done. The process analyses changes and determines how these changes affect emotions and beliefs (particularly opinions) and whether personality (coping preferences) needs to be adapted. In this way the agent's personality in the form of preferred ways of coping, does eventually change depending on the agent's experience. Evaluation uses key variables that relate to the process that has triggered the evaluation, as well as the agent and the environment's state. The variables are measured objectively, however they are combined by using weightings to reflect personality differences that result in placing more or less importance on different variables. This aspect of evaluation and personality is described further in section 3.2.

\section{Emotion and Personality Representation}

Emotions are implemented in line with the OCC method [18], which uses a fixed number of emotions, many of which are paired, e.g. happy and sad. We follow Ortony's [17] suggestion to build an emotional model by beginning with a simple good/bad representation as implemented by, for example, [19]. Each agent has its own emotionality [17] or emotion personality which reflects the fact that emotional events affect different people either more or less and that some people express emotions more readily than others.

Emotionality is expressed in the following values for each individual emotion (ie separate values for positive and negative emotions) which are fixed at agent initialization:

- decay - the rate (can be linear, polynomial or exponential function) at which the emotion goes towards its stable value (e.g. zero) at each time tick;

- uptake - the rate at which a relevant evaluation affects the emotion, i.e. a positive evaluation is multiplied by the positive uptake value to determine 
amount of change in this emotion and vice versa. High uptake values result in an agent that is easily affected by small changes;

- threshold - the value above or below which the emotion is expressed, and the value that triggers changes in coping preferences.

In our framework, personality is explicitly present in emotionality, coping preferences, and evaluation weights. The latter two affect the two appraisal processes: appraisal of coping processes and evaluation of changes respectively.

\subsection{Personality in Coping Preferences}

Coping preferences reflect which plans the agent expects will manage its emotions (or improve its positive emotions) best in a given situation (ie environmental and personal state). An agent's coping preferences, an aspect of its personality, are used by the appraisal of coping strategies process to order which strategies should be tried first. In this case personality reflects the fact that different people use different plans to manage the same emotional event. For instance, when unhappy an introvert may decide to read a book, while an extrovert may decide to go to a party.

The coping preferences of an agent are represented by each input state being one-to-one matched to a list of preference values. The input represents state and emotion variables in a pre-specified order. The list of preference values is ordered so that it is possible to lookup the individual preference value (on a scale of 0-9) for a given plan or coping strategy. The combination of all the input state to preference list pairs make up the agent's coping personality, or more accurately their coping preferences.

As execution progresses the agent encounters new input states, so it randomly allocates initial preferences for these new states. After execution of a coping process the agent performs an evaluation on the process. It may be that, due to environmental or internal changes, the preferred approach no longer produces a desired outcome or produces a much more desirable outcome than expected as determined by the evaluation or re-appraisal process. The ranking of the chosen approach, ie the coping preference for that process, is increased or decreased in two stages. In the first stage, preferences are increased or decreased immediately based on whether the last emotion event is above or below (respectively) the threshold set by the agent's emotionality. In the second stage, preferences can only change after repeatedly bad or good experiences, thus allow for a more stable personality.

Since we include the perceived state of the environment as input to the appraisal of coping preferences process it is possible to get different but consistent behavior across situations. In this case a situation is defined as a specific state of the agent, for example the agent is currently happy and has lots of friends. As a result, if the environment changes and becomes more (or less) adverse the agent changes its coping preferences to match its environment. This process is much easier to implement than tailoring which actions each agent should perform and when. Even though our system is very simple there are already a large amount 
of diverse experiences available for the agent to encounter. Therefore the agent is able to generate its own coping preferences that are suitably diverse compared to the other agents.

\subsection{Personality in Evaluation Weights}

Every time we analyze an outcome we weight certain variables more or less highly than others. For instance, depending on the person an event such as obtaining treasure but no friends may or may not be evaluated as successful. We call the weights or importance of different variables, evaluation weights.

In some ways many evaluation weights could be decided based on analysis of an agent's goals, and beliefs. However, our aim is to implement a simple system. We do not want to expand goals and beliefs to encompass more than necessary. By including evaluation weights we are assigning these values in a simpler, easier to change manner. If all agents had the same evaluation method their coping preferences would eventually converge since they would all be attempting to reach the same states.

In our system the evaluation weights apply to every variable that is used in evaluation, and the sum of the evaluation weights adds to $100 \%$. An overall result from evaluation is obtained by summing the objective results multiplied by their respective weightings. The result or overall success of the triggering event is used to update emotions, opinions (beliefs) about others and the environment and, importantly, to update coping preferences.

It would be awkward to adapt both coping preferences and evaluation weights at the same time since they rely on feedback from each other to make adaptations. In our case we decided to fix evaluation weights after an initialization period. During initialization all agents perform evaluation in the same manner, with even weights on all variables. Based on their initial experience the weights are changed and as such their evaluation personality is fixed for the rest of the execution time.

\section{Future Work, Discussion and Conclusion}

We are currently testing our application based on school children making friends through conversations, as described in section 2.1. Our main aim in testing this application is to determine which parameters are most important in increasing (or decreasing) agent diversity as defined based on agent behavior. Parameters we will examine include: evaluation weights and method of evaluation, coping preferences, emotionality variables, and initialization variables such as number of agents, amount of initial environmental knowledge and more. Our current goal is to vary parameters one at a time to determine their effect on behavior output of the agent population.

To obtain quantitative results of the effect parameters have on behavior we allow the agents to interact in their virtual world and determine whether we can find groups or clusters of agents based on their personality in the form of 
behavioral choices. We are searching for clusters that are significantly different to each other, as well as being stable and not skewed undesirably towards certain personalities. This aims to confirm that the agents have believable and distinct personalities that do not change quickly and without reason. We will allow input parameters to range from all agents being initialized with the same values to all agents being randomly or directly allocated different values. Our future work will concentrate on confirming diverse personalities are obtained and maintained and to determine which parameters are essential to achieve this result. Subsequent work will be to apply the model to a more complex computer game environment using only the essential parameters as found during testing.

There has been increasing use of emotions, personality, and adaptation in virtual characters across a wide-variety of applications. However, prior work does not cover using adaptation for personality development nor apply all these techniques to a game based environment. We will now discuss our work as related to prior work in the field in order to illuminate the gaps that we fill.

Computer game developers are using emotions and learning to compete with other games beyond graphical improvements. For instance, the game Romeo and Juliet [9] allows the player to use a painting canvas to influence the emotions of the two characters, who in turn visualize their emotions with ballet movements. Façade [1] is an emotional interactive drama where the player interacts with a couple who are having serious relationship problems. The crowd generating effects that are able to be produced by Massive software [2] have been very successful. Although this work investigates giving personality to the large number of characters that make up crowd scenes, this work concentrates on superficial animation effects and is yet to be implemented in an interactive environment. Learning is also an area that is being introduced to games, for example Black and White [8], which also implemented BDI techniques. Emotions, personality and learning are not yet widely implemented in games due to perceived high risk and development times required.

A major method of improving virtual characters in the more academic world is to construct them with emotion and personality models. Our use of coping preferences to guide choice of coping strategies or behaviors is similar to $[3,11$, $22]$. However, this past work has relied on emotions only and not personality based on experience to constrain decision-making. One of the most thorough implementations of an emotion model is the work by Gratch and Marsella [10] which also implements beliefs and intentions, however personality and desires are not core concepts. Their model is highly realistic due to their requirement to train military personnel, resulting in more complexity than is required for an entertainment application and their model would be computationally expensive for more than a few characters.

Many implementations of personality are based on trait based approaches from psychology work which do not consider context [16]. That is, it assumes people act in the same manner no matter what the environment and situation. Rousseau and Hayes-Roth [20] attempted to resolve this problem, but then required a substantial amount of set-up time for the designer compared to our 
adaptation method. Almost all implementations of personality assume that it is static, e.g. [16, 20]. This is reasonable, although it assumes that the initial model of personality is sufficiently complex to capture human personality. Our method allows personality to develop according to the environment and become more complex than the initial model if necessary.

A substantial amount of work into emotions and personality requires programmers to devote long times writing pre-scripted behaviors or developing character personalities, e.g. [3,5]. Promising work is being done to automate character behavior and the scripting process (e.g. [7]), so that characters do not follow a fixed script like in a play. This later work indicates that users 'prefer' (find more interesting or are more willing to suspend disbelief) non-scripted characters. However, this work often is only suited to a small number of characters where the personality of characters is hand-crafted.

Our work is different to other work such as [21] that uses emotions to assist the learning or adapatation process since we do not assume that there is an optimum solution or personality, nor do we assume that the user is guiding the agent's choice of what is good and bad. We want agents with diverse behaviors where they have their own optimum solution based on personality that has developed from their unique experience.

In conclusion, emotions and personality are key components of creating believable virtual characters. Current methods of implementing emotions and personality are often too complex or are not freely available to developers of computer games with many virtual characters. In the past personality has usually been implemented in a static, trait-based and hand-crafted manner, leading to long designer development times and characters that are unable to adapt to situation and environment. Our framework applies simplified models of emotions and adaptation to create diverse characters that can develop their personality to suit their environment.

\section{Acknowledgments}

We would like to thank Agent Oriented Software and Ralph Rönnquist for their support under the Planning and Learning in BDI Agents Grant, LP0560702.

\section{References}

1. Façade website by Procedural Arts. http://www.interactivestory.net/.

2. Massive software website. http://www.massivesoftware.com/.

3. E. André, M. Klesen, P. Gebhard, S. Allen, and T. Rist. Integrating models of personality and emotions into lifelike characters. In Affective interactions: towards a new generation of computer interfaces, pages 150-165. Springer-Verlag New York, Inc., New York, NY, USA, 2000.

4. A. Bandura. Social Learning Theory. Prentice-Hall, New Jersey, USA, 1977.

5. H. da Silva Corrêa Pinto and L. O. Alvares. Extended behavior networks and agent personality: Investigating the design of character stereotypes in the game unreal 
tournament. In T. Panayiotopoulos, J. Gratch, R. Aylett, D. Ballin, P. Olivier, and T. Rist, editors, Intelligent Virtual Agents, 5th International Working Confere nce, IVA 2005, Kos, Greece, September 12-14, 2005, Proceedings, volume 3661 of Lecture Notes in Computer Science, pages 418-429. Springer, 2005.

6. A. Damasio. Descartes' Error: Emotion, Reason and the Human Brain. G.P. Putnam, USA, 1994.

7. J. Dias, A. Paiva, and M. Vala. Can users feel sorry for autonomous synthetic characters? In C. Pelachaud, E. André, S. Kopp, and Z. Ruttkay, editors, Proceedings of the Workshop on Creating Bonds with Embodied Conversational Agents (Humanoids), The Fourth International Joint Conference on Autonomous Agents and Multi Agent Systems (AAMAS 2005), July 2005.

8. R. Evans. Varieties of learning. In S. Rabin, editor, AI Game Programming Wisdom. Charles River Media Inc, Hingham, MA, USA, 2002.

9. B. Gilman. Student postmortem: 6mSoft's Romeo and Juliet. Gamasutra, February 2005. http://www.gamasutra.com/features/20050204/gilman_01.shtml.

10. J. Gratch and S. Marsella. A domain-independent framework for modeling emotion. Journal of Cognitive Systems Research, 5:269-306, 2004.

11. S. H. Hemenover and S. Zhang. Anger, personality, and optimistic stress appraisals. Cognition and Emotion, 18(3):363-382, 2004.

12. C. Jones. Chuck Amuck: The Life and Times of an Animated Cartoonist. Farrar, Straus and Giroux, New York, USA, 4th edition, 1994.

13. J. E. Laird. Research in human-level AI using computer games. Communications of the ACM, 45(1):32-35, 2002.

14. R. Lazarus. Emotion and Adaptation. Oxford University Press, USA, 1991.

15. R. R. McCrae and O. P. John. An introduction to the five-factor model and its applications. Journal of Personality, 60:175-215, 1992.

16. D. Moffat. Personality parameters and programs. In R. Trappl and P. Petta, editors, Creating Personalities for Synthetic Actors: Towards Autonomous Personality Agents, volume 1195 of Lecture Notes in Artificial Intelligence, pages 120-165. Springer-Verlag, Germany, 1997.

17. A. Ortony. On making believable emotional agents believable. In R. Trappl, P. Petta, and S. Payr, editors, Emotions in Humans and Artifacts, pages 189-212. MIT Press, Cambridge, MA, USA, 2002.

18. A. Ortony, G. L. Clore, and A. Collins. The Cognitive Structure of Emotions. Cambridge University Press, USA, 1988.

19. L. Padgham and G. Taylor. PAC - personality and cognition: an interactive system for modelling agent scenarios. In Proceedings of International Joint Conference on Artificial Intelligence IJCAI'97, Tokyo, Japan, 1997.

20. D. Rousseau and B. Hayes-Roth. A social-psychological model for synthetic actors. In AGENTS '98: Proceedings of the second international conference on Autonomous agents, pages 165-172, New York, NY, USA, 1998. ACM Press.

21. M. Seif El-Nasr, T. R. Ioerger, and J. Yen. PETEEI: a PET with evolving emotional intelligence. In AGENTS '99: Proceedings of the third annual conference on Autonomo us Agents, pages 9-15, New York, NY, USA, 1999. ACM Press.

22. A. Stern. Creating emotional relationships with virtual characters. In R. Trappl, P. Petta, and S. Payr, editors, Emotions in Humans and Artifacts, pages 333-362. MIT Press, Cambridge, MA, USA, 2002. 